\title{
Tolerable Maximum Positive End Expiratory Pressure in Mechanically Ventilated Patients and Its Impact on Blood Flow across Cardiac Valves: Index Case Report of a Physiology Study
}

\author{
Rashid Nadeem $^{\text {a }}$ Kamaleldin Ahmed ${ }^{\text {b, c }}$ Lamiaa Salama $^{\text {a }}$ Ashraf M. Elhoufi ${ }^{a}$ \\ a Department of Intensive Care, Dubai Hospital, Dubai Health Authority, Dubai, UAE; ${ }^{b}$ Department of Cardiology, \\ Dubai Hospital, Dubai Health Authority, Dubai, UAE; 'Tanta University, Tanta, Egypt
}

\section{Keywords}

Positive end expiratory pressure - Cardiac output . Trans-mitral valve flow · Trans-pulmonic valve flow · Pulmonary flow

\begin{abstract}
Introduction: Positive end expiratory pressure (PEEP) exerts variable effects on preload, pulmonary vascular flow, and afterload. Lung recruitment with gradual increase in PEEP improves oxygenation, but it may have variable cardiovascular effects. Method: A patient with respiratory failure from tetanus was recruited after informed consent. The sedated and paralyzed patient was subjected to sequential increase in PEEP with measurement of hemodynamics, ventilator parameters, and echocardiographic parameters (Doppler flow across tricuspid, pulmonic, and mitral valves at end inspiration and end expiration, in addition to blood volume flowing across RVOT and mitral valve). Results: We observed an initial rise in pulmonic and mitral blood flows, followed by a decline back to baseline around PEEP of 20, with an eventual decline associated with a drop in mean MAP $<60$ torr above PEEP of 29. Similar effects were also observed on
\end{abstract}

\begin{tabular}{ll}
\hline KARGER & ( 2019 The Author(s) \\
& Published by S. Karger AG, Basel Oparger \\
E-Mail karger@karger.com & This article is licensed under the Creative Commons Attribution- \\
www.karger.com/dmj & NonCommercial-NoDerivatives 4.0 International License (CC BY- \\
NC-ND) (http://www.karger.com/Services/OpenAccessLicense). \\
Usage and distribution for commercial purposes as well as any dis- \\
tribution of modified material requires written permission.
\end{tabular}

blood volume flowing across RVOT and mitral valve. PEEP of 5 was associated with best blood flow parameters. Conclusion: Although systemic pressure was maintained at PEEP of 29, pulmonary blood flow started to be compromised at PEEP of 20. Best pulmonary perfusion parameters were achieved at PEEP of 5 .

(C) 2019 The Author(s)

Published by S. Karger AG, Basel

\section{Introduction}

Positive pressure mechanical ventilation is non-physiologic, and PEEP is even more so, but it is required to oxygenate and ventilate patients with respiratory disorders. PEEP affects lung mechanics, oxygenation, and ventilation; it increases functional residual capacity and oxygen stores resulting in improved oxygenation, sometimes at the cost of reduction in ventilation with resultant increase in $\mathrm{PCO}_{2}$ [1]. PEEP affects preload, pulmonary vascular flow, and left heart inflow and outflow. As PEEP increases, right ventricular outflow tends to decrease, reducing blood return to the left ventricle $[2,3]$. Recruit-
Rashid Nadeem, MD, FACP, FCCP, FAASM

Department of Intensive Care Medicine, Dubai Hospital PO Box 7272

Dubai (UAE)

E-Mail rnadeem@dha.gov.ae 
Table 1. PEEP effects on cardiac echocardiographic parameters

\begin{tabular}{|c|c|c|c|}
\hline PEEP & T Vmax Insp. & P Vmax Insp. & M Vmax Insp. \\
\hline 0 & 0.71 & 0.98 & 0.85 \\
\hline 5 & 0.64 & 1.11 & 0.96 \\
\hline 8 & 0.55 & 0.9 & 0.87 \\
\hline 11 & 0.49 & 1.06 & 0.89 \\
\hline 14 & 0.58 & 0.84 & 0.81 \\
\hline 17 & 0.51 & 0.86 & 0.72 \\
\hline 20 & 0.45 & 0.79 & 0.63 \\
\hline 23 & 0.52 & 0.76 & 0.66 \\
\hline 26 & 0.54 & 0.7 & 0.56 \\
\hline 29 & 0.51 & 0.66 & 0.66 \\
\hline PEEP & T Vmax Exp. & P Vmax Exp. & M Vmax Exp. \\
\hline 0 & 0.69 & 1.11 & 0.85 \\
\hline 5 & 0.67 & 1.35 & 0.99 \\
\hline 8 & 0.6 & 0.92 & 1.01 \\
\hline 11 & 0.55 & 1.07 & 0.96 \\
\hline 14 & 0.63 & 0.94 & 0.93 \\
\hline 17 & 0.47 & 0.93 & 0.83 \\
\hline 20 & 0.5 & 0.94 & 0.77 \\
\hline 23 & 0.53 & 0.92 & 0.72 \\
\hline 26 & 0.5 & 0.86 & 0.67 \\
\hline 29 & 0.56 & 0.76 & 0.63 \\
\hline PEEP & $\begin{array}{l}\text { T VTI Insp. } \\
(\mathrm{CW})\end{array}$ & $\begin{array}{l}\text { P VTI Insp. } \\
(\mathrm{CW})\end{array}$ & $\begin{array}{l}\text { M VTI Insp. } \\
(\mathrm{CW})\end{array}$ \\
\hline 0 & 17.4 & 22.8 & 24.7 \\
\hline 5 & 21.4 & 24.8 & 26.1 \\
\hline 8 & 23 & 21.9 & 25.6 \\
\hline 11 & 15.8 & 22.2 & 24.6 \\
\hline 14 & 15.8 & 20.2 & 25.3 \\
\hline 17 & 15.8 & 22 & 23.6 \\
\hline 20 & 17.4 & 18.3 & 20.4 \\
\hline 23 & 20.7 & 18.3 & 19.4 \\
\hline 26 & 18.5 & 14.7 & 19.3 \\
\hline 29 & 20.5 & 15.8 & 16.5 \\
\hline
\end{tabular}

ment maneuvers are safe and effective to improve oxygenation and static compliance in patients with severe acute respiratory distress syndrome. During recruitment maneuvers in acute respiratory distress syndrome, increasing application of PEEP is employed to provide optimal opening of alveoli, which may be at the expense of cardiovascular adverse effects [4].

The maximum amount of tolerable PEEP in normal and diseased lung is a matter of debate. Current practice of recruitment maneuvers mainly aims at the effect of PEEP on oxygenation, ventilation, BP variation, and pulse pressure variation. Its precise impact upon cardiac

\begin{tabular}{|c|c|c|c|c|}
\hline \multicolumn{2}{|l|}{ PEEP } & RVOT SV & \multicolumn{2}{|l|}{ MV SV } \\
\hline 0 & & 69 & 179 & \\
\hline 5 & & 86 & 232 & \\
\hline 8 & & 76 & 185 & \\
\hline 11 & & 78 & 190 & \\
\hline 14 & & 65 & 187 & \\
\hline 17 & & 68 & 182 & \\
\hline 20 & & 65 & 171 & \\
\hline 23 & & 59 & 144 & \\
\hline 26 & & 48 & 136 & \\
\hline 29 & & 45 & 122 & \\
\hline PEEP & MAP & $\begin{array}{l}\text { P VTI Resp. } \\
\text { variation } \\
\text { (Insp.-Exp./ } \\
\text { mean) }\end{array}$ & $\begin{array}{l}\text { Exp. flow } \\
\text { variation of } \\
\text { outlet/inlet } \\
(\text { exp. VTI M/P) }\end{array}$ & $\begin{array}{l}\text { Insp. SV } \\
\text { variation } \\
\text { RVOT/MV }\end{array}$ \\
\hline 0 & 65 & -0.079 & 0.995 & 0.385 \\
\hline 5 & 65 & 0.069 & 1.238 & 0.371 \\
\hline 8 & 66 & -0.01 & 1.158 & 0.411 \\
\hline 11 & 66 & -0.042 & 1.065 & 0.411 \\
\hline 14 & 66 & -0.105 & 1.241 & 0.348 \\
\hline 17 & 70 & -0.146 & 1.189 & 0.374 \\
\hline 20 & 63 & -0.127 & 1.088 & 0.38 \\
\hline 23 & 60 & -0.22 & 1.135 & 0.41 \\
\hline 26 & 60 & -0.182 & 1.253 & 0.353 \\
\hline 29 & 60 & -0.24 & 1.033 & 0.369 \\
\hline
\end{tabular}

functions is largely unknown. We aim to study this effect. Our primary aim was to estimate the maximum pressure of safely tolerable PEEP. Secondary outcomes were to study its impact on blood flow across cardiac valves, cardiac output, stroke volume, and pulmonary inflow and outflow variation during respiratory cycles.

\section{Case Report/Method}

This study was formally approved by the Dubai scientific research ethics committee. The target patient population included patients with indication of mechanical ventilation and without contraindication to apply PEEP with increase in $\mathrm{PCO}_{2}$. Moreover, paralysis should be employed for medical management, not for research study. Comprehensive screening process identified and recruited a 26-year-old male patient who was admitted with tetanus requiring mechanical ventilation and paralysis for tetanus treatment. Informed consent was obtained from the patient's family. Inclusion criteria also required normal cardiac and brain functions. The patient had normal sensorium and cerebral functions when he presented with lockjaw and muscle spasms prior to being mechanically ventilated. Screening echocardiogram showed nor- 


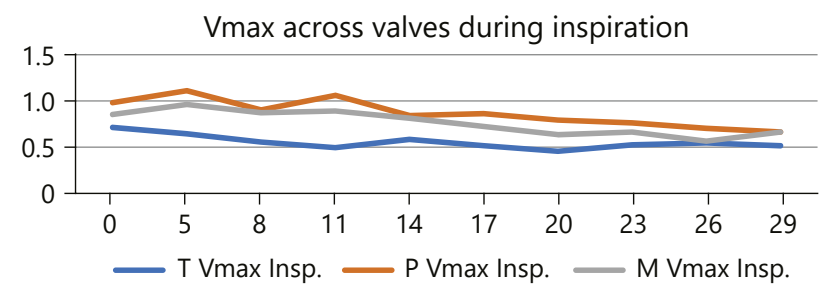

RVOT SV and MV SV

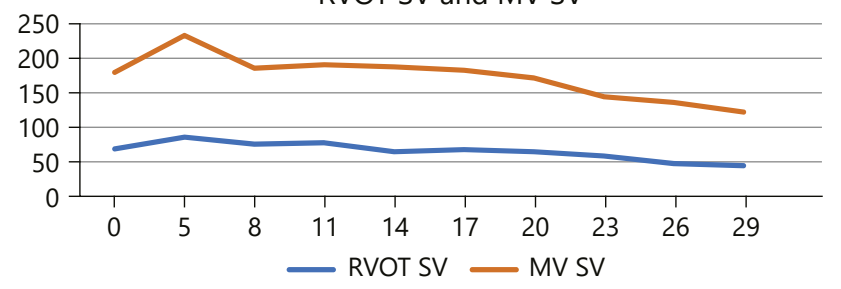

Flow variation, SV variation
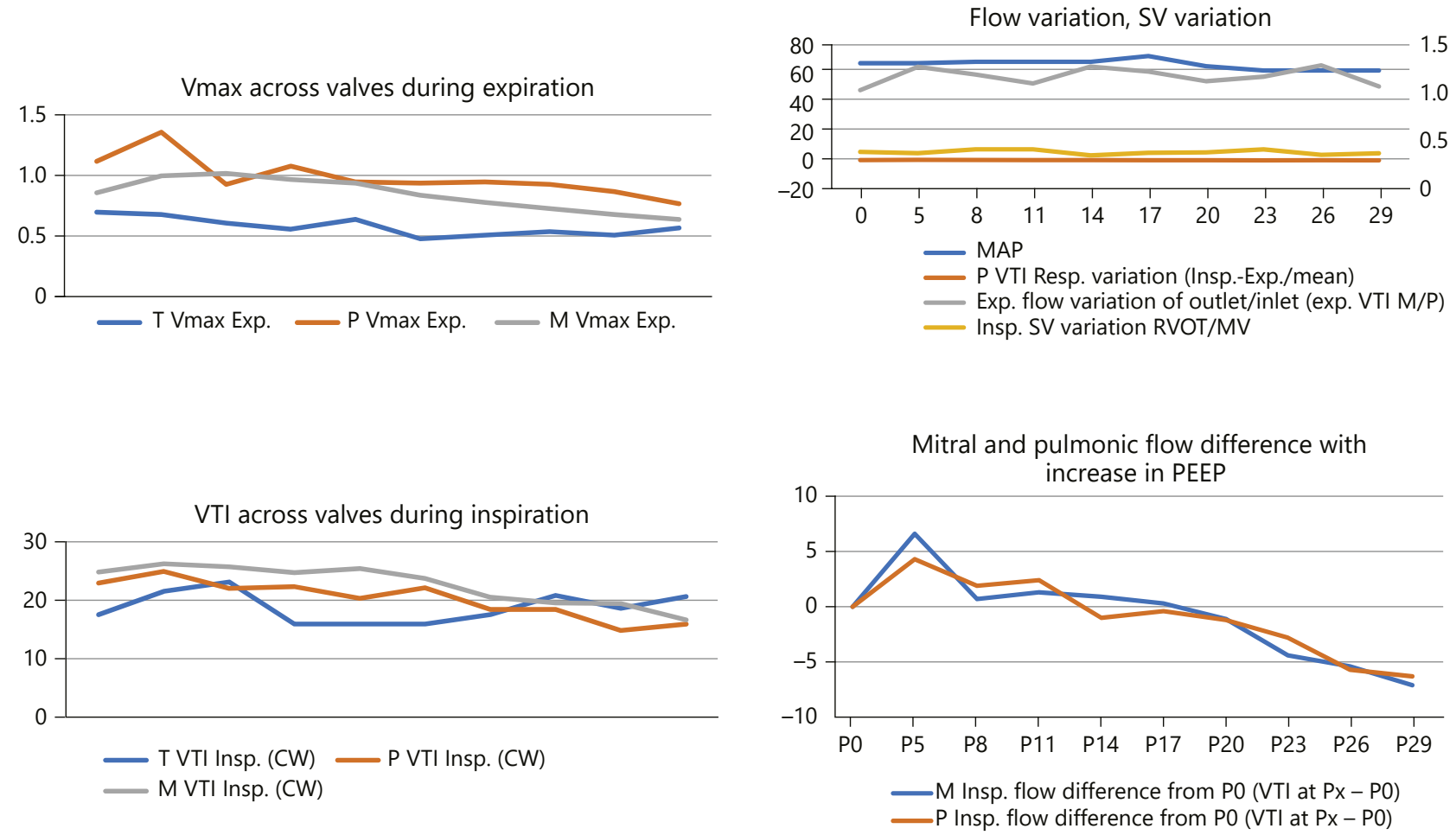

Fig. 1. PEEP and cardiac parameters and their relationships.

mal cardiac function and normal fluid status by measuring inferior vena cava diameter, $\mathrm{BP}$, and pulse pressure variation method from arterial line. The patient was sedated with midazolam and paralyzed with cis-atracurium. Continuous monitoring of hemodynamics (pulse, mean blood pressure, $\mathrm{SPO}_{2}$ ) and respiratory parameters (end tidal $\mathrm{CO}_{2}$, dynamic compliance) were performed. The patient had a fixed tidal volume of $6 \mathrm{~mL} / \mathrm{kg}$ with a respiratory rate of 12 on controlled mechanical ventilation mode.

A consultant certified echo-cardiographer performed all echocardiograms. Baseline as well as repeated echocardiograms were performed with each PEEP level after change starting from PEEP zero, then to 5 , and then after cumulative increase by 3 each time until imminent clinical deterioration; mean arterial BP drop of $>5$ mm Hg or MAP less than 60, as patients MAP was 65. Signs of any physical distress, HR increase $>10$, or significant adverse change in pulmonary/ventilator parameters (low compliance change of 5, high peak pressure $>30 \mathrm{~cm}$ of $\mathrm{H}_{2} \mathrm{O}$, high $\mathrm{EtCO}_{2}>50 \mathrm{~mm}$ of $\mathrm{Hg}$ ). The study was stopped at PEEP $32 \mathrm{~cm}$ of water without any measurement at that level as mean BP dropped to $59 \mathrm{~mm} \mathrm{Hg}$. The patient tolerated PEEP of 29.

For each setting of PEEP, the following cardiac parameters were recorded; tricuspid (T) Vmax during inspiration, $\mathrm{T}$ Vmax during expiration, T VTI Insp. continuous wave (CW), pulmonic (P) Vmax Insp., P Vmax Exp., P VTI Insp. (CW), P VTI Insp. pulsed wave (PW), P VTI Exp. (PW), P VTI Resp. variation (Insp.Exp./mean), mitral (M) Vmax Insp., M Vmax Exp., M VTI Insp. (CW), M VTI Insp. (PW), M VTI Exp. (PW), M VTI Resp. variation (Insp.-Exp./mean), M Insp. flow difference from P0 (VTI at 
Px - P0), P Insp. flow difference from P0 (VTI at Px - P0), Insp. flow variation of pulmonary outlet/inlet (Insp. VTI M/P), Exp. flow variation of outlet/inlet (Insp. VTI M/P), RVOT stroke volume (SV), MV SV, Insp. SV variation RVOT/MV.

\section{Results}

The patient's respiratory, hemodynamic, and echocardiographic parameters are presented in Table 1 and Figure 1. Initially, PEEP increase was associated with increase in pulmonic and mitral flow followed by decrease in flow, back to baseline flow around PEEP of 20 with eventual further decline associated with drop in MAP. PEEP of 29, though well tolerated, was associated with worse flow across these valves than baseline. Biventricular stroke volume also improved at PEEP of 5 followed by gradual and slow decline with further increase in PEEP.

\section{Discussion}

Our study determined that application of PEEP for lung recruitment is safe and tolerated well. Our patient tolerated PEEP of 29, while PEEP of 32 resulted in worsening of MAP without any other detrimental effect. Lou et al. [5] also found that optimal level of PEEP can be safely titrated in traumatic brain injury with close monitoring of cerebral and systemic hemodynamics.

We found that in flow across cardiac valves, there was an initial increase followed by a decrease, with a sequential increase in PEEP. A prospective study of five patients found that a high level of PEEP frequently compromised cardiac index secondary to most likely reduced coronary flow secondary to elevated myocardial interstitial pressure [6]. Another study addressing recruitment maneuvers suggests the risks associated with recruitment maneuvers are both at the ventilator-associated lung injury followed by the systemic risk of hemodynamics impairment or injury to distant organs [7]. Schuster et al. [8] measured hemodynamic parameters and transesophageal pressure simultaneously at PEEP levels $0,4,8,12$, and $16 \mathrm{~cm} \mathrm{H}_{2} \mathrm{O}$ in subjects with heart failure and demonstrated that end-diastolic area of the right atrium decreased significantly while RA pressure increased, and transmural pressure remained unaltered, though the cardiac index decreased. The decrease in cardiac output by PEEP ventilation was related to the decrease in RV filling volume by external compression [8].
Therefore, improvement in oxygenation may be offset by decrease in cardiac output, which results in decreased oxygen consumption. This impact of PEEP is much more complex in patients with structural lung disease or respiratory muscle functions. Acute hemodynamic effects induced by overloading of respiratory muscles were attenuated by addition of PEEP in COPD patients [9]. Clinicians should make an informed and objective determination about the net gain achieved by increase in PEEP.

\section{Conclusion}

Under controlled conditions, higher levels of PEEP are well tolerated. PEEP of 29 appears to be the highest tolerated PEEP in our patient. We noted an initial rise in blood flow across all cardiac valves followed by a gradual decline. Studies are needed to investigate the immediate effect and long-term impact of PEEP on cardiopulmonary parameters and clinical outcomes.

\section{Statement of Ethics}

The authors have no ethical conflicts to disclose. Written informed consent was obtained from the family. There are no patient's identifiers in the case report which may link the patient to the report.

\section{Disclosure Statement}

All authors and their relatives have nothing to disclose, no conflict of interest and no financial interest in the subject matter.

\section{Author Contributions}

Dr. R.N. conceived the idea, wrote the case report, extracted data and analyzed data, and wrote and approved the manuscript. Dr. K.A. conceived the idea, performed ECHO, extracted data, and wrote and approved the manuscript. Dr. L.S. conceived the idea, extracted data, and wrote and approved the manuscript. Dr. A.M.E. conceived the idea, extracted data, and approved the manuscript. 


\section{References}

1 Satoh D, Kurosawa S, Kirino W, Wagatsuma T, Ejima Y, Yoshida A, et al. Impact of changes of positive end-expiratory pressure on functional residual capacity at low tidal volume ventilation during general anesthesia. J Anesth. 2012 Oct;26(5):664-9.

2 Mitaka C, Nagura T, Sakanishi N, Tsunoda Y, Amaha K. Two-dimensional echocardiographic evaluation of inferior vena cava, right ventricle, and left ventricle during positivepressure ventilation with varying levels of positive end-expiratory pressure. Crit Care Med. 1989 Mar;17(3):205-10.

3 van den Berg PC, Grimbergen CA, Spaan JA, Pinsky MR. Positive pressure inspiration differentially affects right and left ventricular outputs in postoperative cardiac surgery patients. J Crit Care. 1997 Jun;12(2):56-65.
4 Póvoa P, Almeida E, Fernandes A, Mealha R, Moreira P, Sabino H. Evaluation of a recruitment maneuver with positive inspiratory pressure and high PEEP in patients with severe ARDS. Acta Anaesthesiol Scand. 2004 Mar;48(3):287-93.

5 Lou M, Xue F, Chen L, Xue Y, Wang K. Is high PEEP ventilation strategy safe for acute respiratory distress syndrome after severe traumatic brain injury? Brain Inj. 2012;26(6):88790.

6 Lozman J, Powers SR Jr, Older T, Dutton RE, Roy RJ, English M, et al. Correlation of pulmonary wedge and left atrial pressures. A study in the patient receiving positive end expiratory pressure ventilation. Arch Surg. 1974 Aug;109(2):270-7.
7 Guerin C, Debord S, Leray V, Delannoy B, Bayle F, Bourdin G, et al. Efficacy and safety of recruitment maneuvers in acute respiratory distress syndrome. Ann Intensive Care. 2011 Apr;1(1):9.

8 Schuster S, Erbel R, Weilemann LS, Lu WY, Henkel B, Wellek S, et al. Hemodynamics during PEEP ventilation in patients with severe left ventricular failure studied by transesophageal echocardiography. Chest. 1990 May;97(5):1181-9.

9 Schaper-Magalhães F, Pinho JF, Capuruço CA, Rodrigues-Machado MG. Positive endexpiratory pressure attenuates hemodynamic effects induced by an overload of inspiratory muscles in patients with COPD. Int J Chron Obstruct Pulmon Dis. 2017 Oct;12:2943-54. 